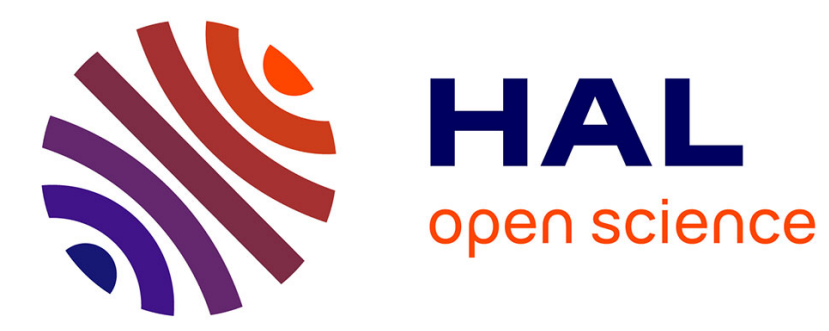

\title{
Une tournée dans les vallées inondées des Alpes Paul Reneuve
}

\section{- To cite this version:}

Paul Reneuve. Une tournée dans les vallées inondées des Alpes. Revue forestière française, 1957, 7, pp.594-598. 10.4267/2042/27333 . hal-03384014

\section{HAL Id: hal-03384014 https://hal.science/hal-03384014}

Submitted on 18 Oct 2021

HAL is a multi-disciplinary open access archive for the deposit and dissemination of scientific research documents, whether they are published or not. The documents may come from teaching and research institutions in France or abroad, or from public or private research centers.
L'archive ouverte pluridisciplinaire HAL, est destinée au dépôt et à la diffusion de documents scientifiques de niveau recherche, publiés ou non, émanant des établissements d'enseignement et de recherche français ou étrangers, des laboratoires publics ou privés. 


\section{UNE TOURNEE DANS LES VALLEEES INONDÉES DES ALPES}

La période du ıo au i6 juin 1957 conservera, dans les annales climatologiques des Alpes, et des Alpes françaises en particulier, par la gravité exceptionnelle des inondations de nombreuses vallées, une bien triste notoriété. La même semaine et la stuivante avaient été, d'autre part, choisies pour le voyage d'études d'économie montagnarde - forêts d'altitude, travaux de lutte contre l'érosion, culture pastorale - de la $129^{\mathrm{e}}$ promotion de l'Ecole Nationale des Eaux et Forêts terminant sa seconde année à Nancy. C'est dire que nos jeunes forestiers purent saisir sur le vif les plus graves conséquences d'excessives précipitations d'altitude et mieux comprendre quelques-uns des périls auxquels sont exposées les populations montagnardes, aussi bien d'ailleurs en belle saison qu'au cours de chaque période hivernale. Ainsi s'expriment au grand jour les rudesses de l'existence des hauts et le danger d'isolement saisonnier, voire total aux temps de catastrophes, qui pèsent si lourdement sur l'homme de la montagne.

Vingt-trois participants, sous la conduite de trois professeurs, parcoururent, du I I au 22 juin, quelque I $300 \mathrm{~km}$ déroulés de Toulon à Grenoble par Nice, Digne, Gap, rayonnant en outre largement à travers les trois départements les plus élevés des Alpes méridionales, dites sèches, ainsi que dans le département de l'Isère. Aux treize Ingénieurs-Elèves de la promotion s'ajoutaient les huit Elèves libres - cinq Libanais, deux Laotiens et un Français - ainsi que deux Ingénieurs forestiers étrangers, en mission d'études à Nancy depuis I956, l'un turc, l'autre chilien.

La presse a fait connaitre que les journées de jeudi I3, vendredi I4 et samedi I 5 furent particulièrement dramatiques, les crues atteignant une puissance inconnue de mémoire d'homme, probablement d'ordre séculaire, bien que d'aucuns aient déjà qualifié l'événement de millénaire.

Dès la soirée du $\mathrm{I} 5$, la caravane forestière atteignait la vallée moyenne de la Durance puis, bientôt, le tronçon inférieur de celle 
du Guil, prenant contact avec une partie de la zone sinistrée, environ la cote mille mètres. Après avoir visité les chantiers de construction du barrage-digue de Serre-Ponçon, échappés de justesse à la pointe de crue durancienne du I4, ils pouvaient observer, au confluent Guil-Durance, la plaine inondée de Montdauphin, le grand pont du Guil effondré, des remblais routiers emportés et la voie ferrée Paris-Briançon coupée puis, un peu plus en amont, les engravements de la route Gap-Briançon en pleine bourgade de la Roche de Rame. Mais l'accès du Queyras restait interdit en raison de la totale disparition, chaussée et tous les ponts, de la route dans la partie supérieure des gorges du Guil sur une dizaine de kilomètres. Il devenait donc impossible de constater tant de douloureuses dévastations de cette " zone-pilote ) d'économic alpine du Queyras,
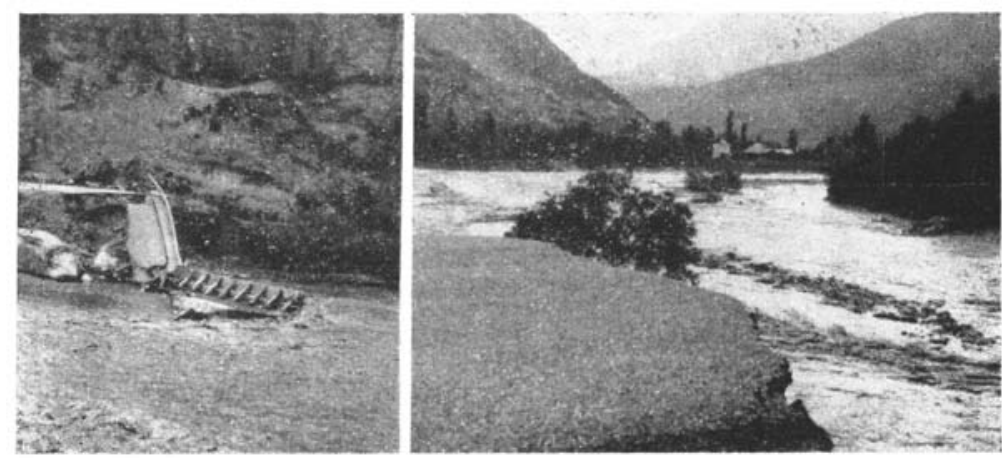

Effondrement d'un pont sur le Guil à l'aval de Guillestre.

(Clichés Boutin.)

objet de tant de soins depuis quelques années pour un équilibre rationnel des productions d'altitude et le maintien des montagnards sur les hauts terroirs; là, de nombreux villages sinistrés, totalement coupés des régions inférieures puisque le col Izoard était également impraticable, payaient leur tribut de misères des habitants des hautes vallées et des reliefs abrupts: lourde dîme échappant, à l'ordinaire discrètement, au citadin chercheur de charmes estivaux et même au sportif coureur de neiges souples sur ıun pan balisé de versant.

Bien d'autres vallées ont été semblablement atteintes au cours de cette même semaine tragique, mais elles restaient à l'écart du périple technique de la $129^{\circ}$ promotion. En haute-Isère, en Maurienne, en Ubave et dans les hautes vallées du $V$ ar et de la Tinée, des flots furieux avaient ravagé les cultures et les habitations, détruit les routes et les ouvrages d'art. C'est l'étendue de la zone montagneuse touchée qui ajoute à l'exceptionnelle gravité des dévastations; du 
Mercantour au Massif de la Vanoise, en passant par le Mont-Viso et les hautes crêtes du Briançonnais, toutes les chaînes alpines frontières ont été incroyablement arrosées plusieurs jours consécutifs. Non seulement la France, mais l'Italie et la Suisse, ont essuyé cette vague d'intenses précipitations.

Peut-on analyser ce phénomène exceptionnel? Il paraît bien prématuré de le tenter. Cependant, les renseignements recueillis sur place et, surtout, complétés par la connaissance des hauteurs d'eau enregistrées dans les postes météorologiques de la zone atteinte,

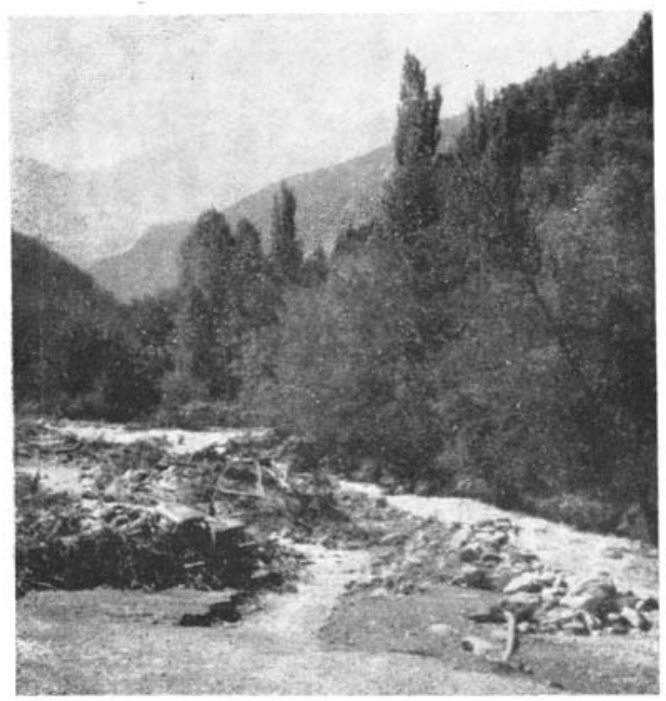

Torrent du Chagne (H.-A.).

La soudaineté de la crue a surpris un conducteur de camion.

(Cliché Boutin.)

permettent de proposer une première explication, évidemment fort peu nuancée, mais qui pourra encadrer une analyse ultérieure complète de l'événement.

D’une façon générale, ce sont les très hautes régions qui paraissent avoir condensé presqu'exclusivement une lame d'eau particulièrement exceptionnelle. Un vent $d u$ Sud-Est dominant a rabattu contre les cimes de la frontière franco-italienne des nuées dont la ( douceur ) était grande: à moyenne altitude, on avait l'impression de recevoir une pluie chaude. C'est ainsi que l'isotherme zéro qui, dans l'ensemble des Alpes françaises du Sud, était à l'altitude 3 roo m le I3 juin, s'est élevé de I $000 \mathrm{~m}$ le I4 (4 IOO $\mathrm{m}$ ) puis, après un léger fléchissement le I5, à $3600 \mathrm{~m}$, a finalement culminé à 
$4300 \mathrm{~m}$ le $\mathrm{I} 6$ juin. Il est donc probable que la plupart des hautes parties arrosées n'ont reçu que de l'eau ou, tout au moins, une très faible partie de neige. D'autre part, les neiges tardives du printemps étaient lourdes et ce qu'il en restait n'a sans doute joué aucun rôle appréciable de rétention; enfin, ce qui en a été libéré par la pluie apparait bien comme de peu d'importance par rapport au volume d'eau précipitée. Les quantités mesurées au cours de la semaine du Io à I6 inclus ont été, en moyenne, pour les postes d'altitude supérieure à 800 mètres, de 2 à 5 fois celles mesurées au cours de la semaine précédente, du 3 au 9 juin. Mais, pour certaines vallées, comme celle de l'Arc en Haute-Maurienne, la proportion ressort à

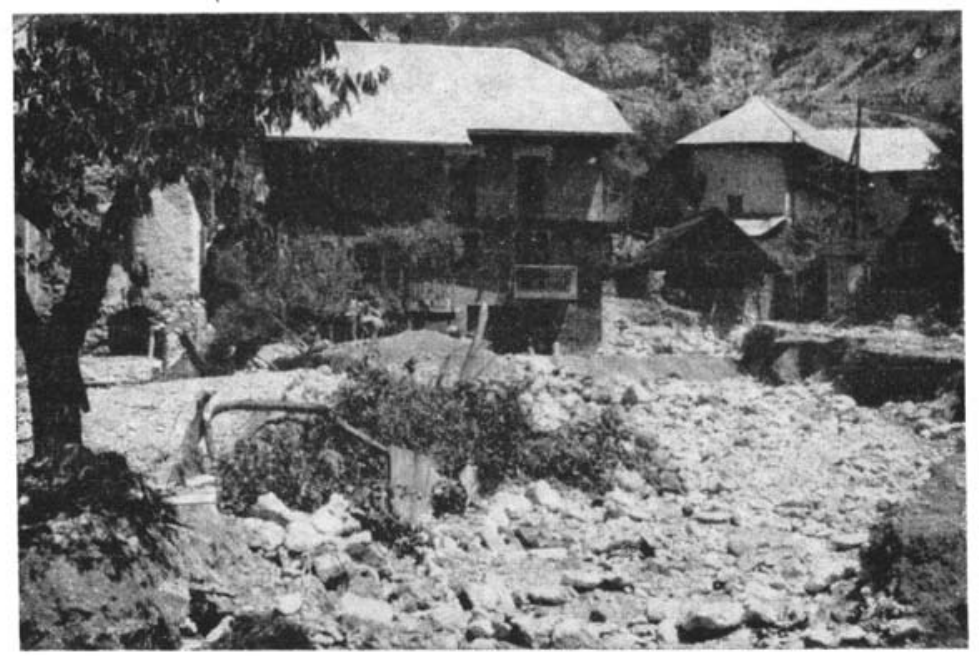

La bourgade de la Roche de Rame (H.-A.) envahie par les déjections du torrent de Bouchouse.

(Cliché Boutin.)

plus de ${ }_{5} 5$ fois, tandis qu'elle se maintient à près de 7 fois pour la Haute-Isère. La tranche d'altitude fortement arrosée est nettement supérieure à I 400 mètres. Plus bas, les valeurs des précipitations diminuent assez rapidement.

Les plus fortes lames mesurées ont affecté, depuis le SudEst vers le Nord-Ouest, les hautes vallées de la Roya et de la Tinée (plus de $100 \mathrm{~mm}$ ), du Var (près de $200 \mathrm{~mm}$ ), du Verdon, de l'Ubaye, de la Durance et de l'Isère (plus de $100 \mathrm{~mm}$ pour chacun des quatre), le haut bassin de l'Arc battant tous les records à ce propos, puisque à Bonneval (à I $700 \mathrm{~m}$ ) $387 \mathrm{~mm}$ ont été totalisés au cours de la semaine du Io au 16 , cependant que la lame d'eau y atteignait $159 \mathrm{~mm}$ pour la seule journée du jeudi 
I3 juin. Or, un peu au-dessous de $\mathrm{I} 500 \mathrm{~m}$, dans la même vallée, le pluviomètre n'a accusé que - si l'on peut dire - I I I mm, durant la même période hebdomadaire.

Les flancs sud-est du Massif de la Vanoise apparaissent donc, jusqu'à présent, comme constituant le pôle des précipitations. On peut, d'autre part, relever quelques variantes: par exemple, le haut Drac n'a reçu, à I $450 \mathrm{~m}$, que $82 \mathrm{~mm}$; ce qui reste explicable par ses hauteurs déjà éloignées, vers l'Ouest, de l'écran condensateur frontière; à l'inverse, le Verdon a recueilli, bizarrement, bien au-dessous de I ooo m d'altitude (Saint-André et Castellane), près de $50 \%$ de plus que ses hautes pentes, c'est-à-dire environ $150 \mathrm{~mm}$. L'isolement à peu près complet de la région du Queyras n'a pas permis de recevoir les données pluviométriques de ses postes; Guillestre, à I $000 \mathrm{~m}$, n'a reçu que $65 \mathrm{~mm}$; il est plus vraisemblable, eu égard aux énormes dommages survenus dans le haut-bassin du Guil, que les précipitations à l'entour du Mont-Viso, ont dû être des plus copieuses.

Il s'agit donc, en bref, d'une précipitation liquide d'une exceptionnelle puissance, venue du Sud-Est, et qui a intéressé toute la zone supérieure intra-alpine mais qui, vers le Nord, n'a pas paru franchir la barrière des massifs centraux Beaufort-Mont-Blanc.

En effet, les bassins de l'Arve et des Dranses n'ont pas accusé, du ro au 16 juin, plus de précipitations que dı 3 au 9 , soit une moyenne de 30 à $35 \mathrm{~mm}$ environ pour chacune de ces deux semaines.

P. Renfuve.

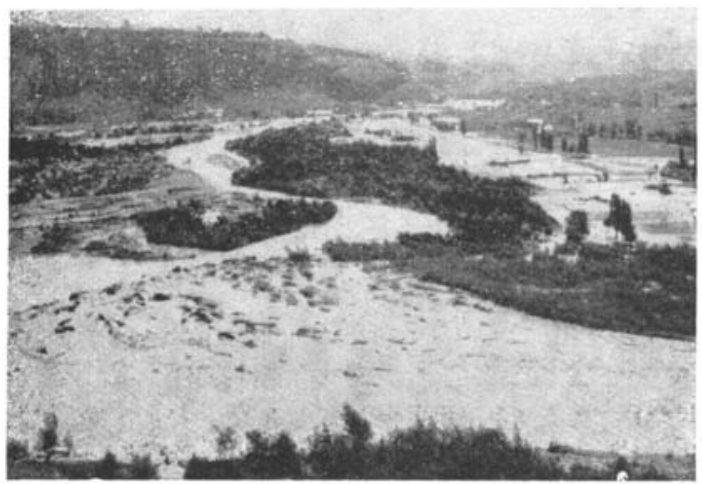

